\title{
Time-Varying Ice Crystal Orientation in Thunderstorms Observed with Multiparameter Radar
}

\author{
I. Jeff Caylor and V. Chandrasekar, Member, IEEE
}

\begin{abstract}
Repeated changes associated with lightning have been observed with multiparameter radar in the echoes from the tops of Florida thunderstorms. These lightning-related radar signatures are interpreted as changes in the orientation of ice crystals being preferentially aligned parallel to the in-cloud electric field. The changes occur at intervals on the order of 10 $s$ and are easily observed in the signatures of the differential propagation phase shift and the linear depolarization ratio which are sensitive to propagation effects caused by the oriented ice crystals. Orientation of ice crystals aloft has been previously observed using circularly polarized radar while the simultaneous differential phase shift and linear depolarization measurements reported here were obtained with a dual-linear polarized radar. The observations indicate crystal orientation angles greater than $45^{\circ}$ and occasionally near vertical. In one case, the crystals were found to be oriented in a layer near radar cloud top spanning a 20-km range and $3 \mathrm{~km}$ in depth.
\end{abstract}

\section{INTRODUCTION}

$\mathbf{V}$ ONNEGUT [1] provided an explanation for abrupt changes observed in the visual appearance of the tops of thunderstorms by suggesting that ice crystals were being oriented preferentially along the electric field. In the subsequent three decades, a number of investigations have been performed dealing with remote sensing of the crystal orientation. Observations of what has been termed anomalous depolarization have been reported in the field of earth-satellite communications [2]-[4]. In general, these results have shown cross talk (cross polarization) between orthogonal polarizations used in earth-space communications channels, often associated with very little fading of the main channel signal. The observed cross polarization and transient effects have been correlated to effects related to microwave propagation through the tops of lightning producing thunderstorms [4], [5].

Among the seminal investigations using microwave radar techniques to investigate this phenomena were those performed using a Canadian $\mathrm{K}_{\mathrm{u}}$-band radar $(1.8 \mathrm{~cm})$ operating with circular polarization. Large regions near the tops of

Manuscript received April 4, 1995; accepted March 8, 1996. This work was supported by the National Science Foundation under Grants ATM-9200761 and ATM-9014600. The work of I. J. Caylor was supported by Goddard Space Flight Center under NASA RTOP 460-24-52.

I. J. Caylor is with the Mesoscale Atmospheric Processes Branch, Laboratory for Atmospheres, NASA/Goddard Space Flight Center, Greenbelt, MD 20771 USA.

V. Chandrasekar is with Colorado State University, Fort Collins, CO 80521 USA.

Publisher Item Identifier S 0196-2892(96)05051-6. thunderstorms containing oriented crystals were observed, whose orientation abruptly changed at the time of lightning [6]. Further investigation [7] produced observations of repeated changes associated with lightning in the phase of the complex correlation of copolar and cross-polar signatures. Using the same $K_{u}$-band radar, a study was performed comparing the orientation of ice crystals in stratiform and convective clouds [8]. Although restricted by a rather short range window of approximately $6 \mathrm{~km}$, these observations produced several instances of crystal orientation changes after a lightning event in the convective clouds.

More recently, interest has revived in this area of radar remote sensing. Krehbiel et al. [9] showed a time sequence of X-band $(3 \mathrm{~cm})$ cross-polar returns at linear polarization and presented evidence that the cyclic nature of the radar signatures is well correlated with electric field variations normally indicative of intracloud lightning. Additional research with the same X-band radar revealed that circular polarization parameters show crystal orientation signatures which provide the potential for prediction of lightning events [10]-[12]. Studies performed at $S$ band $(11 \mathrm{~cm})$ using circular polarization have shown numerous instances of lightning associated crystal alignment in the signature of the cross-covariance amplitude ratio which provides an estimate of propagation effects and scatterer orientation [13]. Although repeated orientation during a given observation period was not clearly observed, it was concluded that the reorientation of ice crystals appeared to be primarily associated with intracloud lightning rather than cloud-to-ground. Recent detailed analysis of the $S$ band radar data has refined methods for estimating orientation angles and revealed ice scatterers with mean canting angles in some cases of up to $90^{\circ}$ just prior to a lightning event [14].

The radar observations presented in this paper were obtained in Florida during the 1991 Convection and Precipitation/Electrification project (CaPE) and clearly show repeated ice crystal orientation with a typical frequency of several times per minute. This paper presents dual wavelength, $S$ and $\mathrm{X}$ band, polarimetric radar observations of the crystal alignment including the first such measurements using the specific differential phase shift $\left(k_{D P}\right)$ between horizontal and vertical linear polarization states. A brief description of the theory of the multiparameter radar measurements as well as theoretical calculations for scattering from ice crystals are presented in Section II. The acquisition of the data is described in Section III and the observations are presented in Section IV. In 
the final section, interpretation of the observations is discussed in terms of propagation effects through the cloud top.

\section{BACKGROUND}

\section{A. Polarimetric Radar Parameters}

The observations were acquired with the National Center for Atmospheric Research CP-2 radar which operates at dual wavelengths of $3 \mathrm{~cm}$ (X band) and $10 \mathrm{~cm}(\mathrm{~S}$ band) with a beam width of approximately $1^{\circ}$ [15]. Horizontal and vertical linear polarizations are used for the polarization diversity measurements. The S-band system is coherent and processes signals copolar to the transmitted pulse which alternates between horizontal and vertical polarization states. The X-band system transmits only vertical polarization but is capable of receiving both the copolar and cross-polar signals. The range gate spacing is $300 \mathrm{~m}$ for both systems. The data processing systems integrate 128 pulses at a pulse repitition frequency of $960 \mathrm{~Hz}$ for each gate, hence a dwell time of $133 \mathrm{~ms}$ between successive estimates for each polarimetric parameter. The CP2 data processor provides a number of polarimetric, parameters and of interest to this work are the reflectivity factor $Z_{S}$ and differential reflectivity $Z_{D R}$ both at $\mathrm{S}$ band and the $\mathrm{X}$-band reflectivity $Z_{X}$ and linear depolarization ratio $L D R$.

The differential reflectivity in units of $\mathrm{dB}$ is defined as

$$
Z_{D R}=10 \log \left(\frac{Z_{H H}}{Z_{V V}}\right)
$$

and is derived from estimates of $Z_{H H}$ and $Z_{V V}$, the copolar reflectivities at $S$ band. The subscripts indicate the state of the transmitted and received polarization, respectively. $Z_{D R}$ is typically used to infer mean shape of the hydrometeors [16]. Since CP-2 is a dual wavelength system we define, $Z_{S}=Z_{H H}$ (S band) and $Z_{X}=Z_{H H}(\mathrm{X}$ band) for notational clarity. The effective reflectivity factor $Z$ has units of $\mathrm{mm}^{6} \mathrm{~m}^{-3}$ and is expressed in logarithmic units of $\mathrm{dBZ}$ by $10 \log (Z / 1$ $\mathrm{mm}^{6} \mathrm{~m}^{-3}$ ).

The linear depolarization ratio (LDR) is estimated from the ratio of the copolar reflectivity $Z_{H H}$ and $Z_{H V}$ the cross-polar reflectivity

$$
\mathrm{LDR}=10 \log \left(\frac{Z_{H V}}{Z_{H H}}\right) .
$$

The LDR signature can be used in a quantitative manner to infer the mean orientation angle of raindrops and more qualitatively to distinguish between wet and dry ice hydrometeors. High LDR values indicate a greater mean canting angle while the lowest LDR indicates either spherical scatterers or symmetrical scatterers aligned in one of the incident polarization planes [17]. LDR is sensitive not only to the canting angle distribution but also to hydrometeor shape, albeit to a lesser degree than $Z_{D R}$.

During the CaPE project, Colorado State University upgraded the CP-2 radar with an auxiliary signal processor which provided several additional S-band polarimetric parameters including the total differential phase shift $\varphi_{D P}$ and the copolar correlation coefficient at zero lag $\rho_{H V}(0)$ [18]. The two-way differential propagation phase shift, $\varphi_{D P}$, is estimated using a pulse-pair algorithm [19]

$$
\begin{aligned}
R_{a}\left(T_{S}\right) & =\frac{1}{M} \sum_{i=0}^{M-1} H_{2 i}^{*} V_{2 i+1} \\
R_{b}\left(T_{S}\right) & =\frac{1}{M} \sum_{i=0}^{M-1} V_{2 i+1}^{*} H_{2 i+2} \\
\phi_{D P} & =\frac{1}{2} \arg \left(R_{a} R_{b}^{*}\right)
\end{aligned}
$$

where $T_{S}$ is the pulse repetition time and $H$ and $V$ are complex valued sample voltages for horizontal and vertical polarization, respectively. The differential phase shift is the total difference in phase between the received horizontal and vertical copolarized waves. At $\mathrm{S}$ band $\varphi_{D P}$ is primarily influenced by propagation effects along the complete path as opposed to backscatter from hydrometeors within a given range gate. For typical meteorological targets, a positive differential propagation phase shift is interpreted to indicate hydrometeors predominantly aligned with their semimajor figure axis along the horizontal. The two-way differential propagation phase shift is defined as the path integral of the specific differential propagation phase shift $\left(k_{D P}\right)$

$$
\phi_{D P}=2 \int_{0}^{r} k_{D P} d r
$$

and so $k_{D P}$, the inherent physical quantity of interest, is estimated by computing the derivative with respect to range of the observed $\varphi_{D P}$. For the observations reported here, the $\varphi_{D P}$ profile is smoothed by a 12 gate running average and the derivative is estimated from a 3-point Lagrangian interpolation. The resulting $k_{D P}$ profile is smoothed with a 9 gate running average.

For a radar such as $\mathrm{CP}-2$ which switches polarization between alternate transmit pulses, the correlation coefficient between the horizontal and vertical received signals is derived from the covariances in (3a) and (3b)

$$
\begin{aligned}
\rho_{H V}\left(T_{S}\right) & =\frac{\left|R_{a}\left(T_{S}\right)\right|+\left|R_{b}\left(T_{S}\right)\right|}{2\left(P_{H H} P_{V V}\right)^{1 / 2}} \\
\rho_{H V}(0) & =\frac{\rho_{H V}\left(T_{S}\right)}{\rho_{H H}\left(T_{S}\right)}
\end{aligned}
$$

where $P_{H H}$ and $P_{V V}$ are estimates of the copolar power [20]. The correlation at zero lag, $(5 \mathrm{~b})$, can be estimated from (5a) and $\rho_{H H}\left(T_{S}\right)$, the autocorrelation of the horizontally polarized signal for a one-pulse lag [21]. The correlation coefficient is sensitive to the distribution of hydrometeor shapes and is also an efficient means to detect lightning intersecting the radar beam.

\section{B. Oriented Ice Crystals}

The theoretical basis for changes of the ice crystal orientation in thunderstorm tops will be briefly reviewed in this section. Although many details of the mechanism responsible for initial separation of charge in thunderstorms are still being debated [22], it is sufficient for the scope of this paper to posit that by the time a cloud reaches a mature glaciated stage the 
charge distribution can be modeled as a dipole structure with (typically) a net positive charge aloft and net negative charge residing lower in the cloud [23]. The resulting dipole structure will tend to produce a vertically oriented electric field in the thunderstorm although the field may deviate from the vertical in localized regions depending on the small-scale distribution of charge.

It is not required that ice crystals carry a net charge to undergo alignment. Calculations have shown that uncharged crystals of up to $800 \mu \mathrm{m}$ can be aligned in the transient fields produced by a charged aircraft moving through the cloud [24]. A detailed physical analysis [25] shows that the conditions existing in a lightning producing thunderstorm are capable of inducing instantaneous dipoles in pristine ice crystals and supporting an out-of-horizontal alignment. Electric fields of $100 \mathrm{kV} \cdot \mathrm{m}^{-1}$ are capable of aligning plates of less than 0.6 $\mathrm{mm}$ diameter and columns of over $2 \mathrm{~mm}$. Larger plates of 1.7 $\mathrm{mm}$ could be aligned by fields of $200 \mathrm{kV} \cdot \mathrm{m}^{-1}$. Fields of $100-200 \mathrm{kV} \cdot \mathrm{m}^{-1}$ are typically found inside thunderstorms although fields occurring at the time of breakdown can be substantially higher [26]. Furthermore, it has been established that in the absence of electric fields in cumulonimbus clouds ice crystals, plates and columns, can be expected to fall with their semimajor axes narrowly distributed around the horizontal [25], [27]. There is a suggestion that alignment of crystals in the horizontal plane with semimajor axes parallel, what is described by Mon [28] as three-dimensional alignment, is even easier to achieve [25]. Turbulence does not significantly disrupt the crystal's general horizontal orientation arising from aerodynamical forces and the crystals will reassume a horizontal orientation in a time on the order of $10 \mathrm{~ms}$ after being released from any orientation [27]. So it can be inferred that if a lightning event should temporarily decrease the strength of the field, electrically aligned ice crystals will quickly assume aerodynamic alignment in the horizontal plane in a time shorter than a radar integration interval which is typically on the order of $100 \mathrm{~ms}$.

Computations have been carried out using the $T$-matrix method to model the differential phase shift at $S$ band for ice crystals. An ice crystal medium with an exponential distribution of size and shape fixed with an axial ratio of 5 was used to model the CaPE radar measurements. Fig. 1 shows the specific differential propagation phase shift $k_{D P}$ and the reflectivity factor for distributions of needles with $D_{o}$ of $1,1.25$, and $1.5 \mathrm{~mm}$ (density of $0.9 \mathrm{~g} \mathrm{~cm}^{-3}$ ). Fig. 2 shows similar results of the scattering calculations for plates with $D_{o}$ of $1,1.25$, and $1.5 \mathrm{~mm}$. The figures show that $k_{D P}$ of $0.2-1.0^{\circ} \mathrm{km}^{-1}$ can be achieved and compare well with estimates indicating $k_{D P}$ of $\pm 0.5^{\circ} \mathrm{km}^{-1}$ in ice [29] and radar observations of ice giving $0.25-0.5^{\circ} \mathrm{km}^{-1}$ [30]. The computed $\mathrm{X}$-band LDR is below $-48 \mathrm{~dB}$ for the crystal distributions shown in Figs. 1 and 2.

The signatures expected for LDR and $k_{D P}$ for a medium composed of oriented crystals can be predicted in a general sense. In the absence of an electric field, the crystals will be loosely aligned under the influence of gravitational and aerodynamic forces with horizontal semimajor axes. As the ambient electric field in the thunderstorm increases, the crystals will be

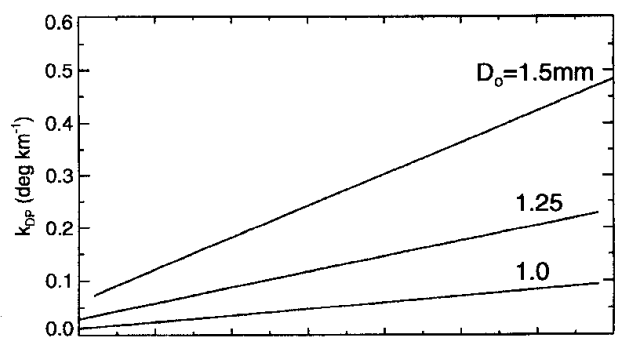

(a)

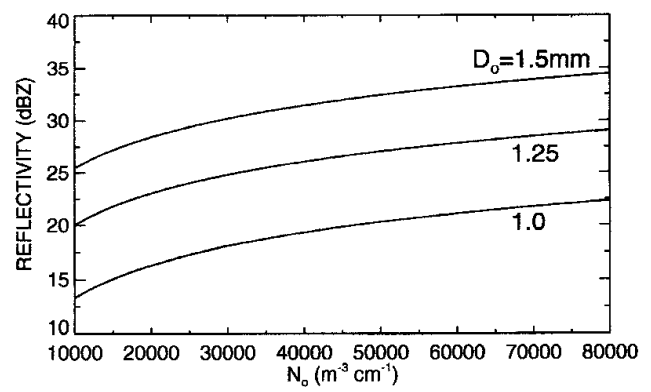

(b)

Fig. 1. Calculated (a) $k_{D P}$ and (b) $Z_{S}$ for an exponential size distribution of ice columns with a fixed axial ratio of 5 . Curves for $D_{o}$ of 1.0, 1.25, and $1.5 \mathrm{~mm}$ are shown.

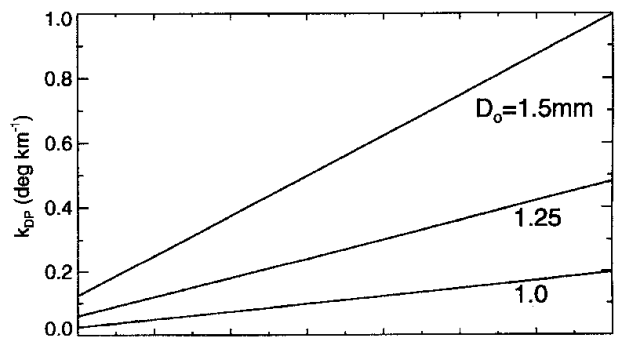

(a)

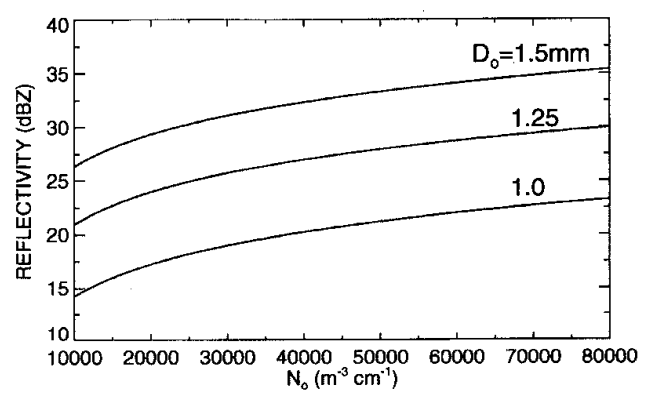

(b)

Fig. 2. As in Fig. 1, except for plates with an axial ratio of 5.

rotated with their major axis aligned parallel to the field lines. Smaller crystals will be easier to align while larger crystals will only be aligned in the strongest fields. Using such a scenario, LDR and $k_{D P}$ will exhibit distinctly different signatures for a medium of aligned crystals as shown in Table I. For an aligned ensemble of scatterers, the largest LDR is associated with a 
TABLE I

Relation of LDR AND $k_{D P}$ TO CRYSTAL ORIENTATION ANGLE

\begin{tabular}{|c|c|c|c|}
\hline & \multicolumn{3}{|c|}{ ORIENTATION } \\
\hline & $0^{\circ}$ & $45^{\circ}$ & $90^{\circ}$ \\
\hline LDR & Min & Max & Min \\
\hline$k_{\mathrm{DP}}$ & Pos & Zero & Neg \\
\hline
\end{tabular}

$45^{\circ}$ orientation where cross polarization is maximized whereas the smallest observable LDR occurs at vertical or horizontal alignment. In contrast, $k_{D P}$ will progress from a positive value associated with horizontal alignment to a negative value at vertical alignment. The transition from positive to negative $k_{D P}$ will occur at the $45^{\circ}$ orientation. This relationship between $k_{D P}$ and depolarization has been observed with signals from a linearly polarized $19-\mathrm{GHz}$ satellite beacon [3].

\section{DESCRIPTION OF THE EXPERIMENT}

During the $1991 \mathrm{CaPE}$ project, the NCAR CP-2 radar was located on the eastern coast of Florida, approximately $20 \mathrm{~km}$ north of Kennedy Space Center (latitude $28.7544^{\circ} \mathrm{N}$, longitude $\left.80.7747^{\circ} \mathrm{W}\right)$. The time-dependent observations presented in Section IV were obtained with the radar in a fixed pointing mode during which the antenna was held at constant elevation and azimuth angles. Thus, data were recorded for a fixed location in space over an extended period of time. No effort was made to continuously slew the antenna to track storm advection. Rather, after a period of time, typically five to ten minutes, the antenna position was updated if it was judged that the active region of the cloud had advected out of the beam. This decision was accomplished by observing changes in the weather echo on the A-scope or with information from a second radar.

Selecting a suitable antenna position for the fixed dwells was done in coordination with personnel operating the New Mexico Institute of Mining and Technology (NMIMT) polarimetric radar [12] which was located $17 \mathrm{~km}$ to the southeast of $\mathrm{CP}-2$. The newly developed display capability of the NMIMT Xband radar allowed for detection of regions of potential crystal alignment in real time. In instances when an active region was found by the NMIMT radar, its location was converted from NMIMT to CP-2 polar coordinates and the CP-2 antenna position was updated accordingly.

Sector scans, either PPI or RHI, were interspaced with the fixed dwells to provide periodic information on the overall storm structure and position. The scans could be viewed on the real-time display whereas no display of processed data (other than the A-scope) was available for the fixed dwells. The observations reported below were made on storms at greater than $60 \mathrm{~km}$ range and so no reliable electric field data are available from the field mill network at Kennedy Space Center.

\section{OBSERVATIONS}

Observations of Florida thunderstorms were collected by the upgraded CP-2 radar on six days in 1991: September 20-22, 25,28 , and 29. Approximately $2 \mathrm{~h}$ of data were collected in the fixed dwell mode alone. The research objective was to perform multiparameter radar observations of the lightning channel and over 200 lightning events were obtained. During postprocessing of the lightning results, though, the more subtle ice crystal orientation signatures became evident.

It must be noted that not every case where the lightning channel was observed with the radar was also associated with an ice crystal alignment signature. This could be potentially because i) the specific region of the cloud where aligned ice crystals are present is not in the radar resolution volume or ii) no observable alignment of crystals occurred. However, crystal orientation signatures in $k_{D P}$ were observed at various times on all six days at slant ranges from $40-125 \mathrm{~km}$ and heights between $7-14 \mathrm{~km}$ (all above the $0^{\circ} \mathrm{C}$ level) which suggests that crystal alignment is a relatively common phenomenon in the Florida thunderstorms. For the purpose of discussion, this paper will focus on observations from September 20-21, 1991 which display most clearly the general features of the ice crystal orientation signatures.

\section{A. Polarimetric Radar Signatures}

Fig. 3 shows time series of the measured parameters observed on September 20, 1991 over a period of $300 \mathrm{~s}(11.9 \mathrm{~km}$ height, $114 \mathrm{~km}$ range). Fig. 3 shows that, of the polarimetric parameters described in Section II, only the total differential phase shift $\left(\varphi_{D P}\right)$ and the cross-polar reflectivity $\left(Z_{H V}\right)$ are seen to be sensitive to crystal alignment. Seven reorientation events related to lightning are seen in these two traces. The differential phase shift changes by up to about $10^{\circ}$ while $Z_{H V}$ can change by $15 \mathrm{~dB}$ following lightning. The errors for these measurements are typically approximately $1 \mathrm{~dB}$ for $Z_{H V}$ and $2^{\circ}$ for $\varphi_{D P}$.

The remaining four parameters, $Z_{S}, Z_{X}, Z_{D R}$, and $\left|\rho_{H V}(0)\right|$, do not exhibit any discernible responses to hydrometeor realignment. The sharp transients seen in the $Z_{S}$ and $\left|\rho_{H V}(0)\right|$ time series are caused by the lightning channel intersecting the pulse volume [31]. The long-term decreasing trend in the reflectivity is probably related to the advection of the thunderstorm through the beam. $Z_{D R}$ is near zero and $\left|\rho_{H V}(0)\right|$ is high, near 0.98 ; both are typical values for ice crystals [32], [33].

In all except one of the seven events ( $t=95 \mathrm{~s}$, Fig. 3), $Z_{H V}$ can be seen to slowly decrease as the electric field builds and after lightning decreases the ambient electric field the crosspolar reflectivity rapidly increases. For all events though, $\varphi_{D P}$ gradually decreases and then abruptly increases after lightning.

A noticeably different situation is shown in Fig. 4. The data were acquired at a height of $10.8 \mathrm{~km}$ at 22:43:36 UTC on Sept. 20, 1991. Again, among the six polarimetric parameters only $Z_{H V}$ and $\varphi_{D P}$ indicate sensitivity to the orientation. In Fig. 4, however, the cross-polar reflectivity exhibits the opposite behavior to that of Fig. 3 with $Z_{H V}$ gradually increasing prior to a lightning event and then decreasing by $5 \mathrm{~dB}$ or less, afterward. The deviations in $\varphi_{D P}$ follow the same pattern as Fig. 3, decreasing prior to lightning followed by a sharp rise. The change in $\varphi_{D P}$ is less than $5^{\circ}$ which, as explained later, can be attributed to a shorter path containing oriented ice crystals. 

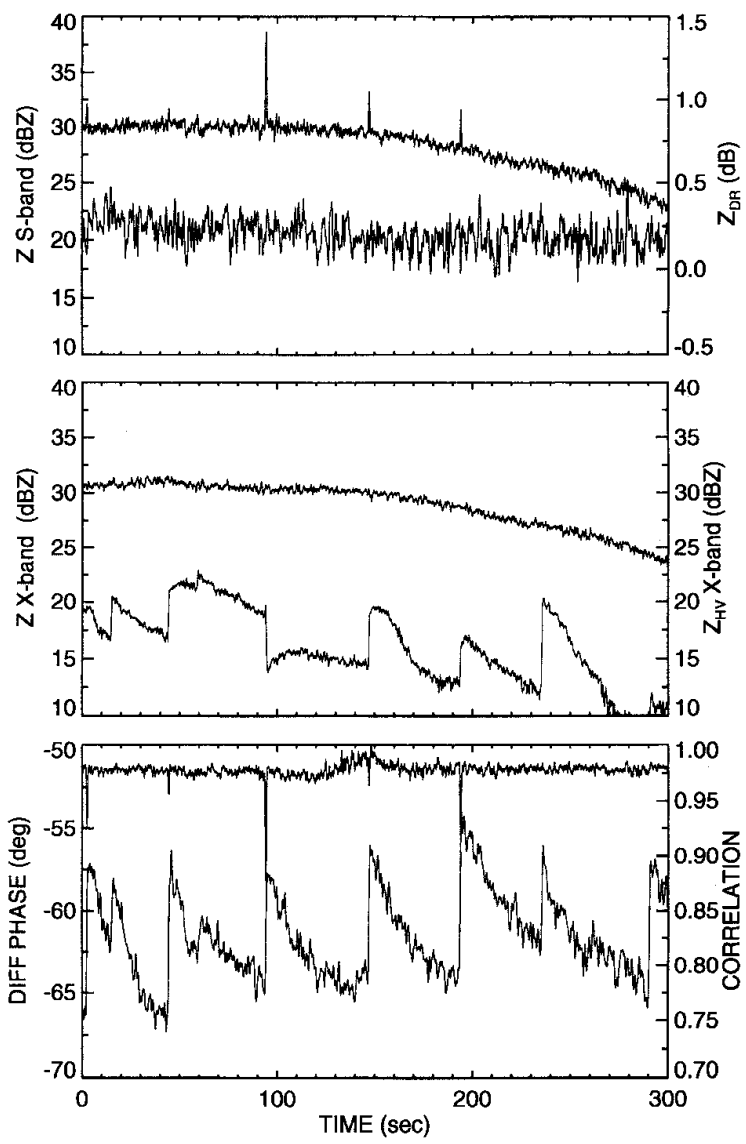

Fig. 3. Time series of the six polarimetric parameters on September 20,1991 at 22:59:59 UTC showing crystal orientation signatures in $\varphi_{D P}$ and $Z_{H V}$. The slant range is $114 \mathrm{~km}$ and the height is $11.9 \mathrm{~km}$. The transients in $Z_{S}$ and $\left|\rho_{H V}(0)\right|$ at times of 95,145 , and $195 \mathrm{~s}$ are due to backscatter from lightning channels. In each panel, the lower trace corresponds to the right axis.

These time-series data clearly indicate that only $\varphi_{D P}$ and $Z_{H V}$ are sensitive to the orientation of the crystals and so the following discussion will focus primarily on these two parameters. As described in the Section II, the specific differential phase shift $\left(k_{D P}\right)$ can be derived from $\varphi_{D P}$ while the linear depolarization ratio (LDR) is derived from the copolar and cross-polar reflectivity. Figs. 5 and 6 show the $k_{D P}$ and LDR time-series derived from the data presented in Figs. 3 and 4, respectively.

The LDR signature (Figs. 5 and 6) mirrors the crosspolar signal quite closely, given that there is no orientation information in the copolar data. However, since $Z_{H V}$ is effectively normalized by $Z_{H H}$, long term trends caused by advection or large scale changes are removed from the LDR signature. $k_{D P}$ is the slope of $\varphi_{D P}$ in range and so the radar system phase is removed from $k_{D P}$. The deviations in $k_{D P}$ compared between Figs. 5 and 6 are now much more comparable, effectively removing differences in the two cases caused by different path lengths. LDR and $k_{D P}$ rather than $Z_{H V}$ and $\varphi_{D P}$ lend themselves somewhat more readily to intercomparison between different cases in the discussion of crystal orientation.
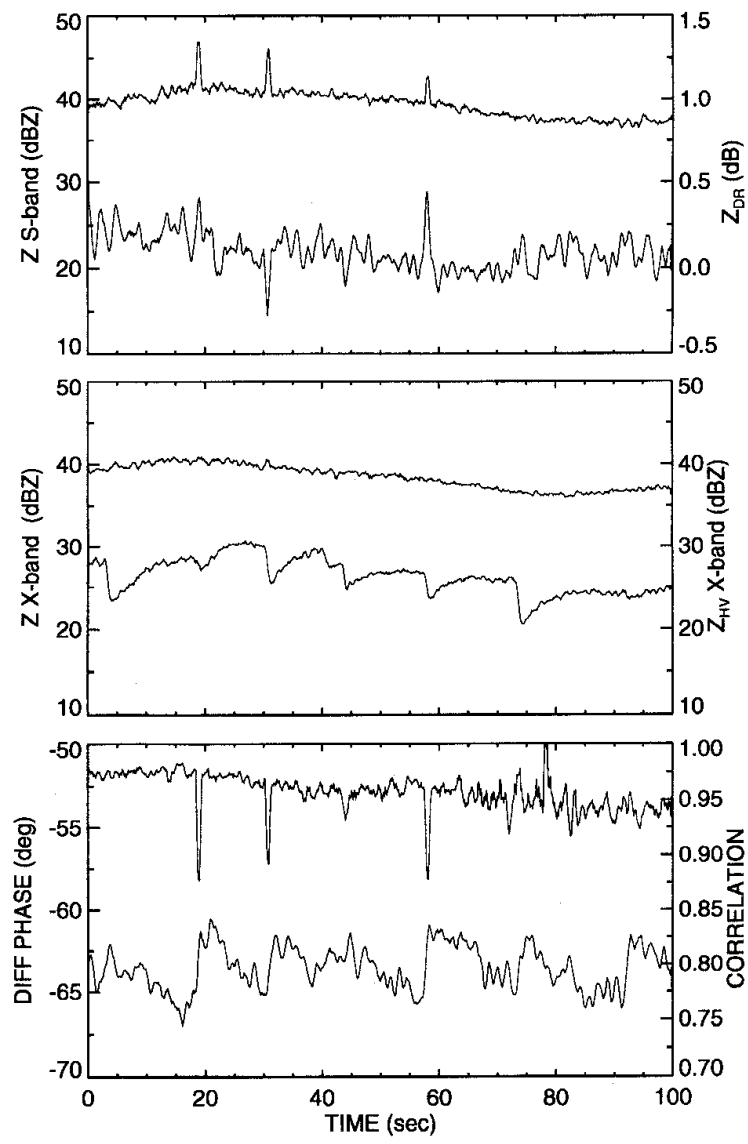

Fig. 4. Time series of the six polarimetric parameters on September 20, 1991 at 23:43:36 UTC showing crystal orientation signatures in $\varphi_{D P}$ and $Z_{H V}$. The slant range is $62 \mathrm{~km}$ and height is $10.8 \mathrm{~km}$. The transients in $Z_{S}, Z_{D R}$, and $\left|\rho_{H V}(0)\right|$ at times of 18,30 , and $57 \mathrm{~s}$ are due to backscatter from lightning channels intersecting the resolution volume. In each panel, the lower trace corresponds to the right axis.

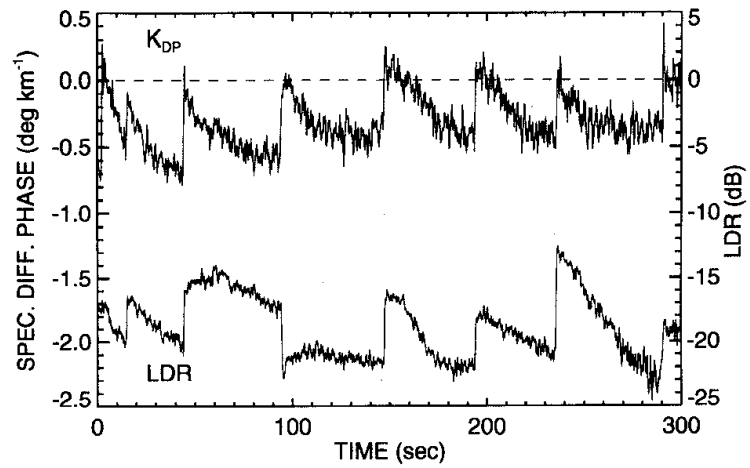

Fig. 5. Time series of $k_{D P}$ and LDR on September 20, 1991 at 22:59:59 UTC derived from the data shown in Fig. 3.

\section{B. Range Dependence}

It is observed that much larger $\varphi_{D P}$ deviations are frequently found in clouds at farther distances. In addition, $Z_{H V}$ transitions related to crystal orientation were often, though not always, larger at greater ranges. A possible explanation for these qualitative observations is that at greater ranges, the 


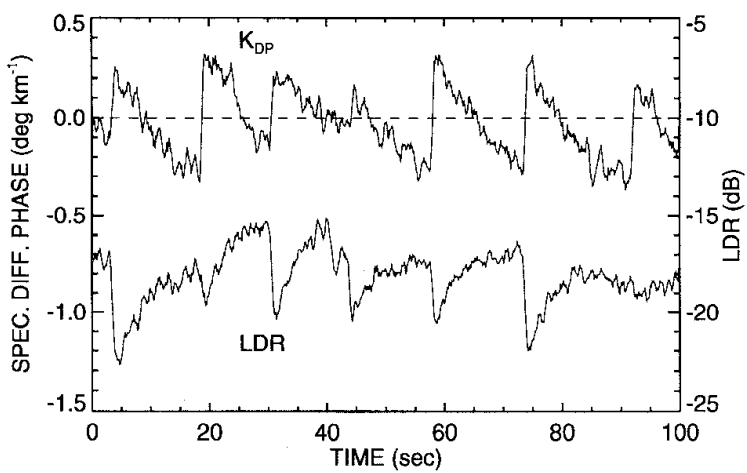

Fig. 6. Time series of $k_{D P}$ and LDR on September 20, 1991 at 23:43:36 UTC derived from the data shown in Fig. 4.

antenna elevation angle is smaller and the more horizontal view often results in a longer propagation path through the cloud top. At closer ranges, although the returned echo power may be greater, the elevation angle is larger for a view of the cloud top and a more oblique look angle results in a shorter path through the oriented crystals which will reduce propagation-related signatures.

Fig. 7 illustrates the propagation dependent nature of the $Z_{H V}$ and $\varphi_{D P}$ signatures. The range profiles immediately before and after a lightning event on September 21, 1991 are shown in Fig. 7(a) where the time interval between the two profiles is $0.94 \mathrm{~s}$. Prior to lightning, the decreasing $\varphi_{D P}$ with range is rather uncommon for clouds, in that it indicates a propagation medium of hydrometeors with a greater mean vertical than horizontal cross section. After the event, the $\varphi_{D P}$ profile assumes a near zero slope while $Z_{H V}$ increases by several $\mathrm{dB}$. (The truncation of the $Z_{H V}$ profile is a result of thresholding against cross polar received power.)

The difference in the two $\varphi_{D P}$ profiles and the two $Z_{H V}$ profiles is plotted in Fig. 7(b). Both difference profiles exhibit a reasonably uniform increase as a function of range which would be expected from a propagation effect. The slope of the $\varphi_{D P}$ profile between ranges $108-126 \mathrm{~km}$ is $0.97^{\circ} \mathrm{km}^{-1}\left(k_{D P}\right.$ is half this) and slope for $Z_{H V}$ is about $0.16 \mathrm{~dB} \mathrm{~km}^{-1}$. For the observed value of $0.16 \mathrm{~dB} \mathrm{~km}^{-1}$, it would take about $5 \mathrm{~km}$ to generate a cross polarization large enough to be observed (approximately $1 \mathrm{~dB}$ ) above the statistical fluctuations in $Z_{H V}$ which can account for the lag of $Z_{H V}$ compared to $\varphi_{D P}$.

Figs. 8 and 9 display the $k_{D P}$ data as a function of range over the previously discussed time periods (Figs. 3 and 4). The $k_{D P}$ scale has been chosen to enhance the negative going $k_{D P}$ (positive $k_{D P}$ omitted) with darker shades indicating greater mean crystal alignment. The figures show the spatial distribution of aligned ice crystals as a function of range and time; the time-series in the previous figures are a leftto-right cut through the range-time image. Fig. 8 indicates a slightly enhanced peak $k_{D P}$ in the first $150 \mathrm{~s}$ compared to Fig. 9 although there is a larger area of oriented hydrometeors in Fig. 8. A larger area of orientation is anticipated for the 22:59:59 UTC observations by the $10^{\circ} \varphi_{D P}$ deviations (Fig. 3) compared to the $5^{\circ}$ and smaller deviations at 23:43:36 UTC (Fig. 4). Figs. 8 and 9 indicate the general spatial nature

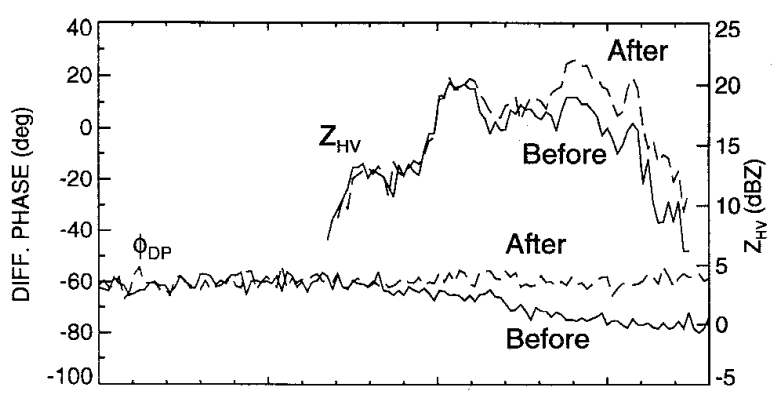

(a)

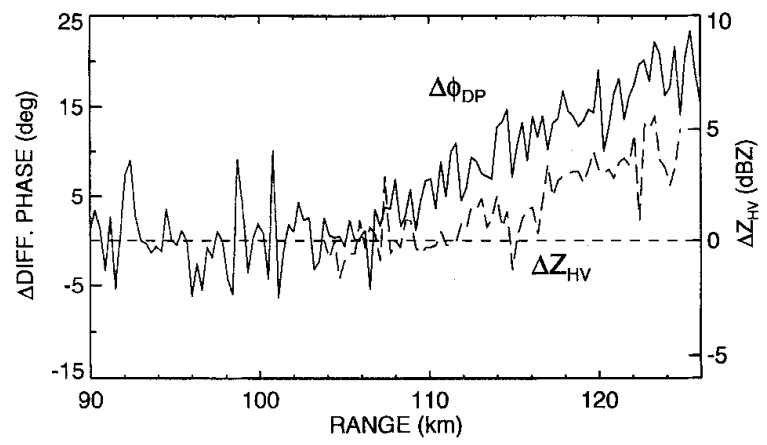

(b)

Fig. 7. Range profiles of (a) $\varphi D P$ and $Z_{I I V}$ immediately before and after a lightning event at 00:36:53 UTC on September 21, 1991. The time interval is $0.94 \mathrm{~s}$ and the antenna elevation is $5^{\circ}$. The difference profiles (b) show the increase of $\varphi D P$ and $Z_{H V}$ as a function of range, more clearly illustrating a propagation effect

of the observed crystal orientation in that following a lightning event, the $k_{D P}$ values become smaller, and in cases turn positive, (e.g., Figs. 5 and 6) indicating a lower degree of orientation. Thereafter, the area of negative $k_{D P}$ expands in range while $k_{D P}$ decreases to more negative values as the electric field recovers resulting in a higher degree of crystal alignment. However, there are cases when the change in crystal orientation is quite localized. For instance, in Fig. 8 at 150 $\mathrm{s}$, the negative $k_{D P}$ area completely disappears at $114 \mathrm{~km}$ range following a lightning event while the negative $k_{D P}$ near $106 \mathrm{~km}$ range is only slightly changed in terms of both range extent and value, indicating the lightning induced change in the electric field was localized.

\section{Reflectivity Dependence}

A reflectivity contour for $25 \mathrm{dBZ}$ is shown in Fig. 8 and for $30 \mathrm{dBZ}$ in Fig. 9. The negative $k_{D P}$ indicating regions of aligned crystals is often centered over the reflectivity maximum, or convective core. A region of aligned crystals is also present outside the convective core at $105-109 \mathrm{~km}$ in Fig. 8; and in Fig. 9, a pair of $k_{D P}$ minima are initially visible near the edges of the reflectivity core from time 0 to $100 \mathrm{~s}$. Since the anvil aloft often blows off downwind of the convective core it is conceivable that areas of crystal orientation can be separated from a reflectivity maximum. However, the $k_{D P}$ minima on the edges of the convective core are intriguing. From the data available it is not possible 


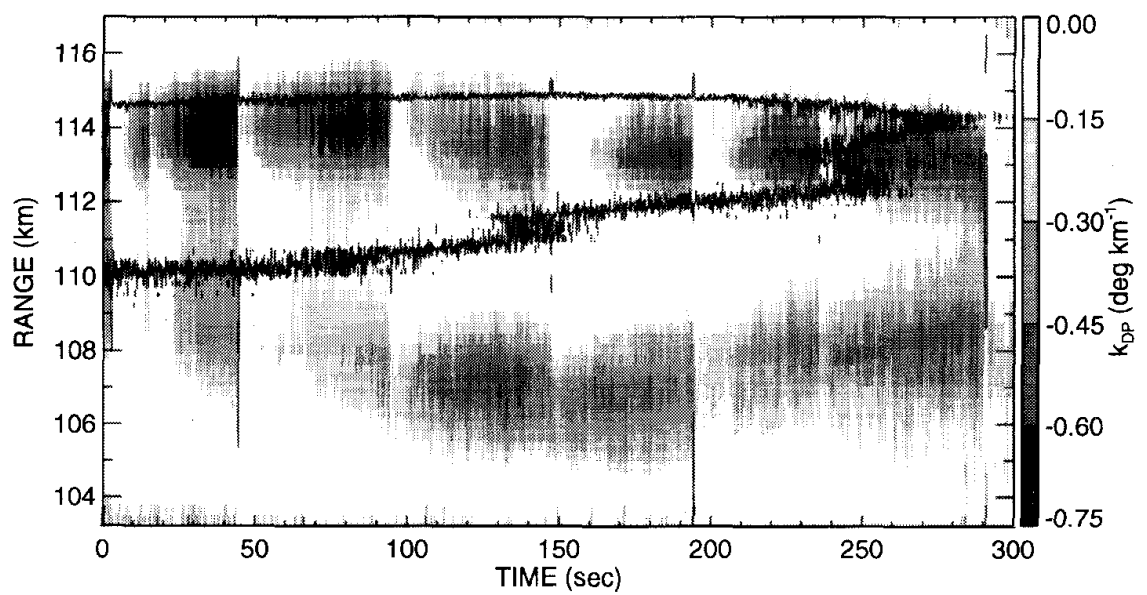

Fig. 8. Range-time image of $k_{D P}$ on September 20, 1991 at 22:59:59 UTC (see Figs. 3 and 5) displaying the spatial distribution of oriented ice crystals over time. The center height of the image is $11.5 \mathrm{~km}$. The scale has been chosen to enhance negative values of $k_{D P}$ associated with crystal orientation. The solid line encloses a region where reflectivity is greater than $25 \mathrm{dBZ}$.

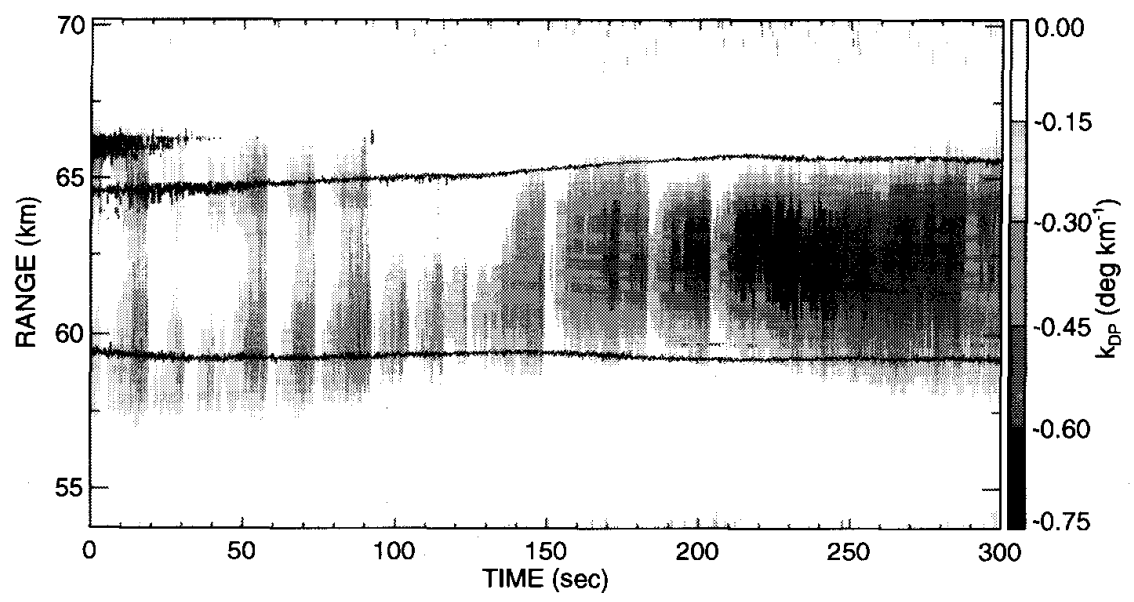

Fig. 9. Range-time image of $k_{D P}$ on September 20, 1991 at 23:43:36 UTC (see Figs. 4 and 6 ). The center height of the image is 10.8 km. The solid lines enclose reflectivity greater than $30 \mathrm{dBZ}$.

to determine in what manner the electrical and hydrometeor characteristics changed after the first $100 \mathrm{~s}$ (Fig. 9). We can speculate from the range-time images that the weak minima could appear in a plan view (PPI) as a ring of oriented crystals around the convective reflectivity core.

No consistent results were found from storm to storm relating the appearance of oriented crystals to a specific reflectivity threshold. Among the observations from these Florida storms, the regions of enhanced negative $k_{D P}$ often appeared to be associated with reflectivity of less than about $35 \mathrm{dBZ}$.

\section{September 21, 1991 Case}

The September 21, 1991 case provides an interesting comparison with the data from September 20. The September 21 thunderstorm (00:32:33 UTC) was more active with 16 reorientation events being observed within a five minute period (Fig. 10). There were also several crystal reorientations that

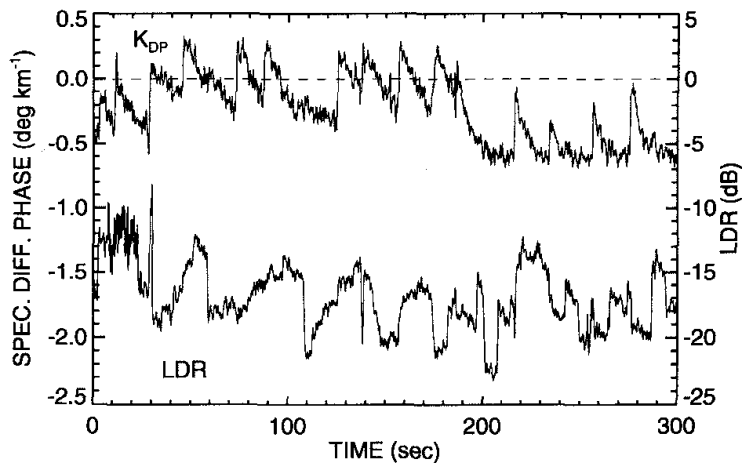

Fig. 10. LDR and $k_{D P}$ time series from September 21, 1991 at 00:32:33 UTC. The range is $117 \mathrm{~km}$ and height $12.2 \mathrm{~km}$. The change in the $k_{D P}$ level at approximately $185 \mathrm{~s}$ is caused by a decrease in the antenna elevation changing the height to $10.4 \mathrm{~km}$.

are seen in LDR but not in $k_{D P}$. These are located at 50 , 105, and $200 \mathrm{~s}$ in Fig. 10. 


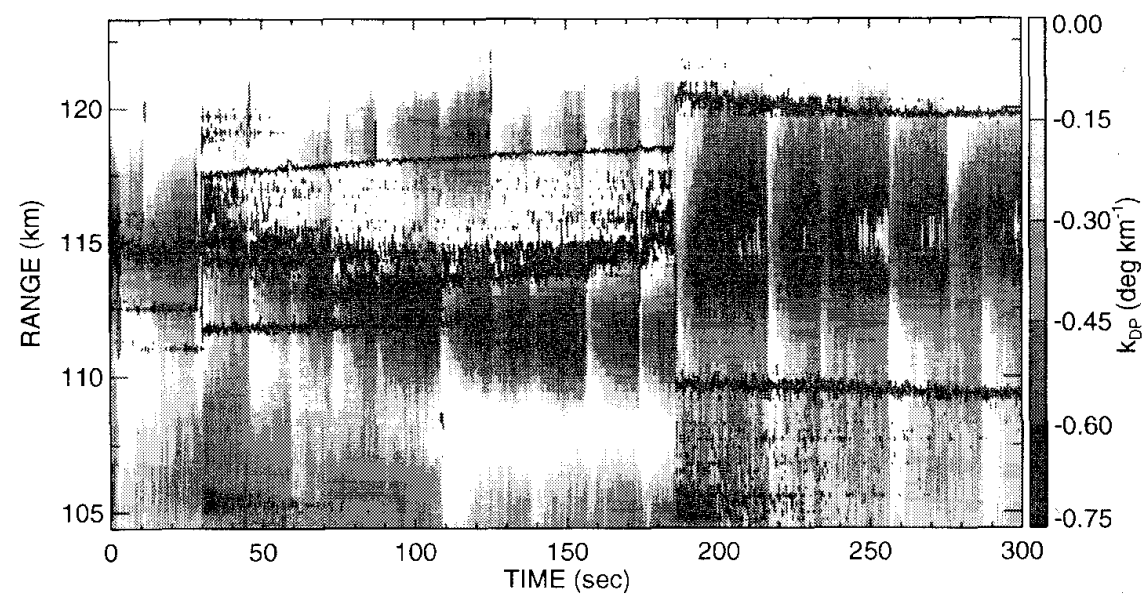

Fig. 11. Range-time image of $k_{D P}$ on September 21, 1991 at 00:32:33 UTC (see Fig. 10). The center height is $11.9 \mathrm{~km}$ and the solid lines are a $29 \mathrm{dBZ}$ reflectivity contour.

The largest negative $k_{D P}$ associated with oriented crystals observed during the field project was recorded on this day. Fig. 11, a range-time image of $k_{D P}$ at $12.2 \mathrm{~km}$ height, shows values of $-0.9^{\circ} \mathrm{km}^{-1}$ at an elapsed time of $250 \mathrm{~s}$ and $115 \mathrm{~km}$ range. At $185 \mathrm{~s}$ there was a $1^{\circ}$ decrease of the antenna elevation angle and Fig. 11 indicates that the beam was moved down into a more extensive region of negative $k_{D P}$. In this region, there was a total range of approximately $20 \mathrm{~km}$ containing oriented crystals.

A contour enclosing the $29 \mathrm{dBZ}$ region is plotted over the $k_{D P}$ observations in Fig. 11. The reflectivity maximum is not well defined during the first $185 \mathrm{~s}$ resulting in a somewhat noisy contour. However, it is clear that the regions of crystal orientation are several kilometers displaced from the peak reflectivity during this time. After the antenna is repositioned, the area of intense $k_{D P}$ is well correlated with the high reflectivity region.

Just prior to the commencement of data acquisition in the dwelling mode, an RHI sector scan was performed at 00:31:28 UTC. Fig. 12 shows a scan taken at $262^{\circ}$ azimuth with decreasing elevation. The three panels show the S-band reflectivity, $k_{D P}$ and $\left|\rho_{H V}(0)\right|$ with reflectivity contours of $15,25,35$, and $45 \mathrm{dBZ}$ overlaid on the $k_{D P}$ and $\left|\rho_{H V}(0)\right|$ images. The $k_{D P}$ scale has been selected to enhance the small negative values and so areas of large positive $k_{D P}$ typically associated with rain at lower altitude are not shown. A region of oriented crystals $3-4 \mathrm{~km}$ deep spanning $20 \mathrm{~km}$ along the cloud top is clearly visible in the $k_{D P}$ field. The two regions of negative $k_{D P}$ are situated slightly away from the peak reflectivity core.

Fig. 13 shows the next scan of the sequence at $263^{\circ}$ azimuth approximately $6 \mathrm{~s}$ later with the antenna scanning upwards from ground level. As the beam passed through the $8 \mathrm{~km}$ height at $110 \mathrm{~km}$ range, lighting occurred and the channel intersected the beam. This event is seen as only a small enhanced reflectivity region and negative $k_{D P}$. However, the lightning channel is clearly visible in the $\left|\rho_{H V}(0)\right|$ image.

Meteorological targets such as rain and snow typically have $\left|\rho_{H V}(0)\right|$ greater than 0.95 while observations of the lightning channel have shown mean values less than 0.8 [31]. It should be noted at this point that the images have been created by interpolating the polar radar data onto a cartesian grid and, as a result, the lightning signature is broadened somewhat. As the antenna elevation continues to increase through the rest of the RHI, it is clear that the region of oriented crystals from $108-120 \mathrm{~km}$ range has disappeared and that $k_{D P}$ values in the remaining area are significantly reduced. The third RHI of the sequence, another $6 \mathrm{~s}$ later at $264^{\circ}$, (Fig. 14) shows a negative $k_{D P}$ region once again spanning the $20 \mathrm{~km}$ range but with reduced values as compared to Fig. 12.

The three RHI scans shown in Figs. 12-14 span a distance of approximately $4 \mathrm{~km}$ in the azimuthal direction which is approximately the smallest radius of a region of oriented crystals shown in Fig. 11. We can conclude that although the scans are cutting through slightly different regions of the storm, they do show the spatial arrangement and lightning related reorientation of a layer of ice crystals in the cloud top. The various observations presented in this section show distinct similarities in that the $Z_{S}, Z_{X}, Z_{D R}$, and $\left|\rho_{H V}(0)\right|$ are free of indications of crystal orientation while $\varphi_{D P}$ and $Z_{H V}$ are quite sensitive to electrically induced orientation effects. Furthermore, the $k_{D P}$ signatures in the three storms are all quite similar indicating ice crystals being pulled into alignment with the vertical electric field. The differences in the LDR signatures will be discussed in the next section.

\section{DISCUSSION}

The salient features of the observations have been identified in the previous section. The various combinations of $k_{D P}$ and LDR signatures will now be considered in terms of a simple conceptual model of ice crystal orientation. At wavelengths of 3 and $10 \mathrm{~cm}, \varphi_{D P}$ is predominantly influenced by propagation effects for a scattering medium consisting of ice crystals while LDR may be interpreted in terms of either backscatter or propagation. Had the cross polarization been a result of backscattering, deviations of 5-15 dB in the cross-polar return from a medium of highly aligned hydrometeors would have an 


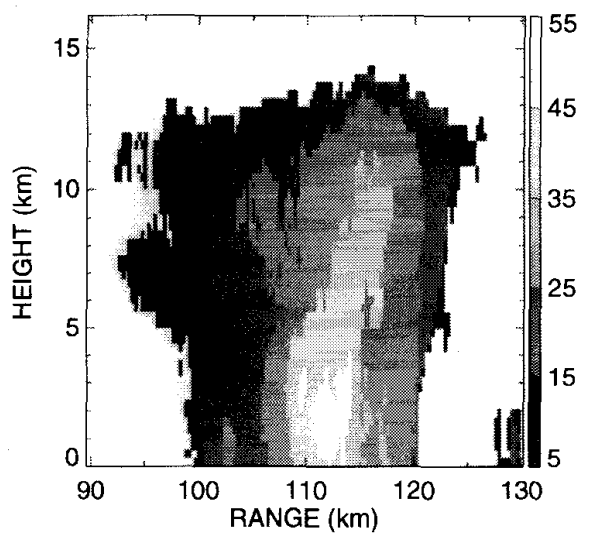

(a)

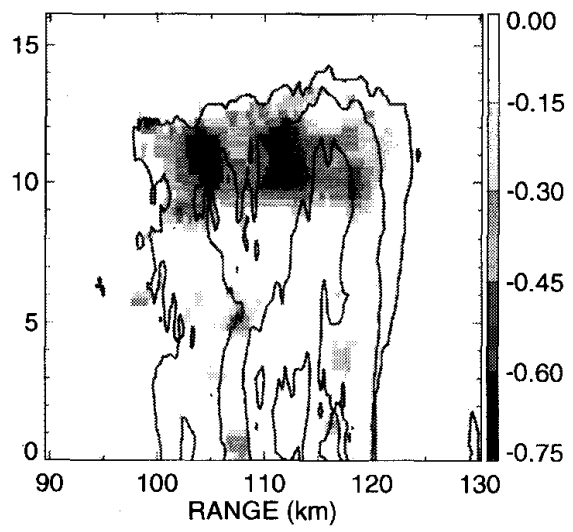

(b)

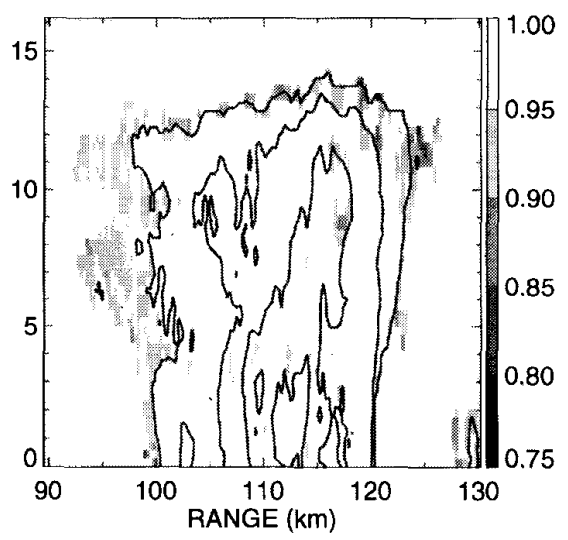

(c)

Fig. 12. RHI scan at $262^{\circ}$ azimuth on September $21,1991,00: 31: 28$ UTC showing (a) $Z_{S}$, (b) $k_{D P}$, and (c) $\left|\rho_{H V}(0)\right| . Z$-contours in (b) and (c) are at $15,25,35$, and $45 \mathrm{dBZ}$. Note the layer of oriented crystals at 9-12 km height indicated by the negative $k_{D P}$.

observable result on the copolar reflectivity $Z_{X}$. However, for a wave with a polarization state different from the preferred direction of alignment of the scatterers in the propagation path, a significant cross-polar component can be created as the wave propagates through the medium. In terms of the differential reflectivity, negative $Z_{D R}$ has been reported for ice aloft in continental thunderstorms [34], but no similar observation was

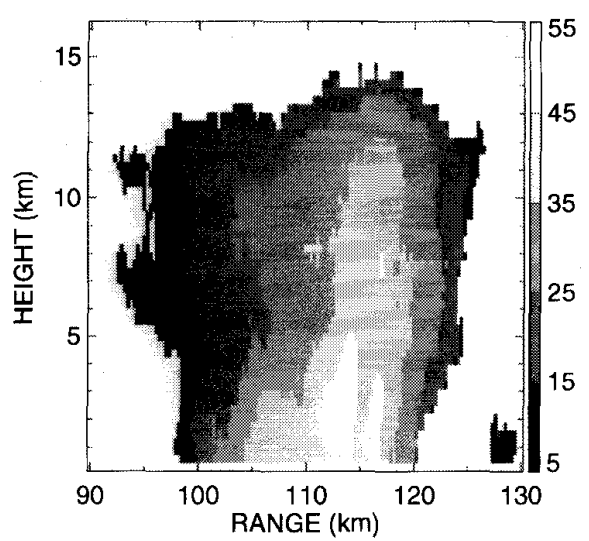

(a)

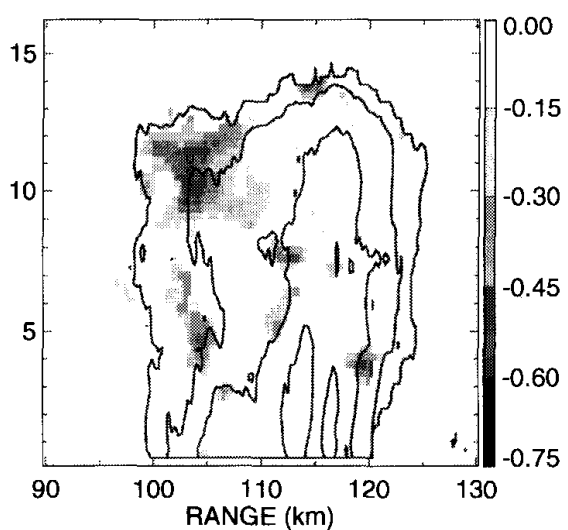

(b)

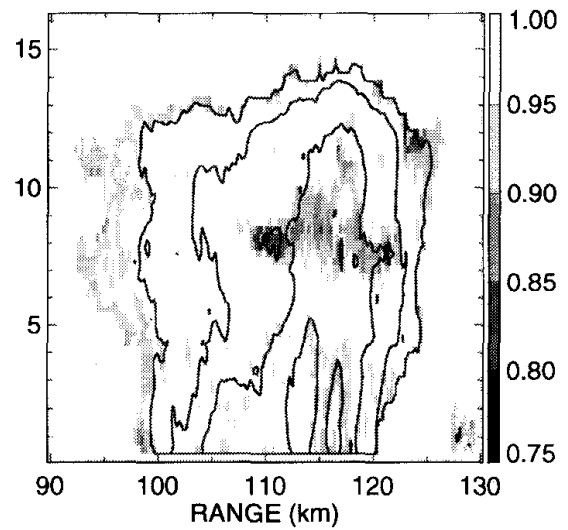

(c)

Fig. 13. RHI scan at $263^{\circ}$ azimuth on September $21,1991,00: 31: 35$ UTC. Lightning is visible in the $\left|\rho_{H V}(0)\right|$ image at $7-9 \mathrm{~km}$ height. The antenna elevation was increasing and the layer of oriented crystals near cloud top has partially disappeared (compare with Fig. 12).

made from the Florida CaPE data. It is worth noting, that on the CP-2 radar LDR is measured at $X$ band $(3 \mathrm{~cm}$ wavelength) while the $Z_{D R}$ measurement is made at the longer wavelength of $10 \mathrm{~cm}$ which is far less sensitive to propagation effects. Being a copolar measurement, $Z_{D R}$ is relatively insensitive to cross polarization along the propagation path compared to LDR and it would be expected that if the LDR signatures were 


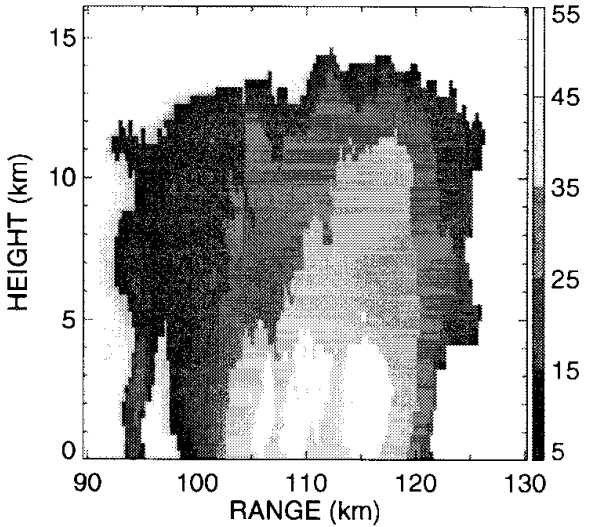

(a)

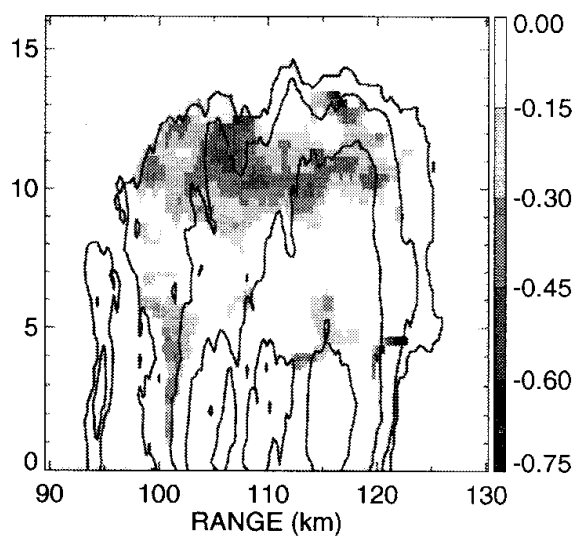

(b)

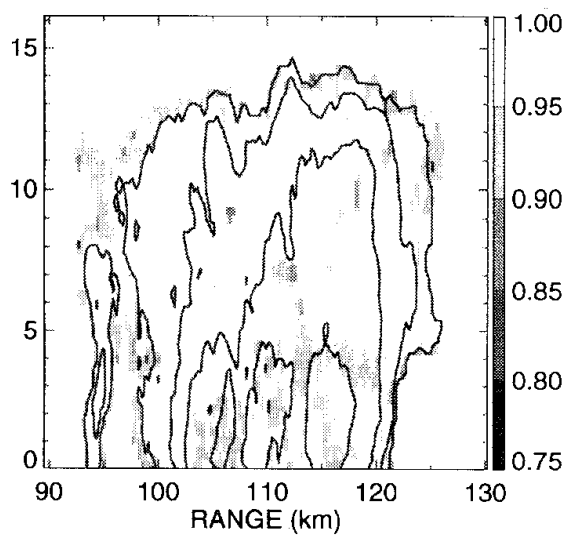

(c)

Fig. 14. RHI images at $264^{\circ}$ azimuth on September 21, 1991, 00:31:41 UTC. The layer of negative $k_{D P}$ (oriented ice crystals) again spans the 20 $\mathrm{km}$ cloud top although slightly weaker than 12 s earlier (Fig. 12).

a result of backscattering there would have been an observable signature in $Z_{D R}$ as well. For the observations presented here it is concluded that the propagation effect dominates the LDR signature. And when the effect of propagation is considered, the various signatures identified in Section IV can be interpreted in a fairly simple, self-consistent manner as described below.
1) Increasing LDR, decreasing $k_{D P}$ prior to lightning. This case can be seen in Fig. 6 and is easily explained by the conceptual model of orientation (Section II). For example, at $20 \mathrm{~s}$ (Fig. 6), there is horizontal alignment with $k_{D P}$ of $0.3^{\circ} \mathrm{km}^{-1}$ and LDR at a minimum. Approximately $5 \mathrm{~s}$ later $k_{D P}$ crosses through zero and LDR nears a maximum indicating an alignment closer to $45^{\circ}$ as the crystals are gradually pulled into alignment with the electric field. After this point, $k_{D P}$ briefly continues to go negative while LDR levels off until lightning occurs and the crystals reassume horizontal alignment.

2) Decreasing LDR, decreasing $k_{D P}$ prior to lightning. The three events at 150, 195, and $235 \mathrm{~s}$ in Fig. 5 are clear examples and again easily explained by the model. In these three events, $k_{D P}$ initially has values near $0^{\circ} \mathrm{km}^{-1}$ while $\mathrm{LDR}$ is at a maximum which implies orientation angles near $45^{\circ}$. As the electric field builds $k_{D P}$ continues negative reaching values near $-0.5^{\circ} \mathrm{km}^{-1}$ and LDR decreases (cross polarization is reduced) as the crystals continue to align more vertically, perhaps closer to $90^{\circ}$.

3) Decreasing LDR, decreasing $k_{D P}$ prior to lightning. Negative LDR step after lightning. This effect is displayed in the section of Fig. 5 between 45-95 s. Prior to the lightning, the crystal orientation can be described by case 2) of crystals near the $45^{\circ}$ orientation being pulled more vertical. However, after the lightning event, $k_{D P}$ returns to zero while LDR drops by $5 \mathrm{~dB}$. An examination of the range-time image (Fig. 8) shows that at the time of lightning (95 s), there was a large decrease in the area of negative $k_{D P}$ along the path which is seen as an increase in the white area between $108-114 \mathrm{~km}$. In this case, $k_{D P}$ is reflecting a local change while LDR is responding to effects along the path. The lightning event changed the orientation of crystals along a large section of the path significantly reducing the cross polarization and dropping LDR, essentially setting a new "baseline."

4) LDR orientation signature with no associated $k_{D P}$ deviation.

This case is shown by the two large LDR deviations at 60 and $110 \mathrm{~s}$ in Fig. 10. The $k_{D P}$ timeseries shows negligible deviations at these times. What is important is that the total differential phase shift, $\varphi_{D P}$, did indeed drop at these times. This is another situation where a lightning event produced a negligible local effect but a significant one at some other point along the propagation path. The range-time image (Fig. 11) indicates, most clearly at $110 \mathrm{~s}$, that a decrease in orientation occurred along a significant portion of the path, particularly $105-110 \mathrm{~km}$, thereby reducing cross 
polarization and LDR. Locally at $117 \mathrm{~km}$, though, there was little response in $k_{D P}$.

5) $k_{D P}$ deviation with little or no associated LDR signature.

This situation appears to be the least common combination of $k_{D P}$ and LDR. Two examples are shown at 72 and $85 \mathrm{~s}$ in Fig. 10. The $k_{D P}$ deviations are centered around $0^{\circ} \mathrm{km}^{-1}$ indicating only slight deviations from horizontal of the crystal orientation. There is only a small change in LDR at these two times. Inspection of Fig. 11 for these times shows $k_{D P}$ is relatively unchanged in the ranges $105-115 \mathrm{~km}$ while a more significant change in $k_{D P}$ occurs at $117-120 \mathrm{~km}$ (the timeseries in Fig. 10 is for $117 \mathrm{~km}$ ). Recall that $k_{D P}$ is the slope of $\varphi_{D P}$. What essentially is happening in this case is that there is a localized change in crystal orientation over a small enough propagation path to leave LDR relatively unchanged.

The various relationships of $k_{D P}$ and LDR signatures at a given range gate are explained by differences caused by electrically induced effects in the propagation properties for the "local area" versus properties over the total path. These variations are a consequence of the use of partial linear polarization data and less ambiguous results can be obtained with full matrix measurements (e.g., circularly polarized radar) [12], [14].

The recovery time for horizontal realignment following a lightning event was estimated for $k_{D P}$ and LDR to be approximately 7 integration cycles or about $1 \mathrm{~s}$. However, the $k_{D P}$ and LDR data are filtered and this lengthens the times somewhat. Examination of the raw $\varphi_{D P}$ data indicates settling times more on the order of about $0.3-0.5$ s ( $2-4$ integration cycles) which correspond well with previous measurements of response times of less than $0.5 \mathrm{~s}$ [3], [9]. Mean flash rates for the cases discussed here are $1-4 \mathrm{~min}^{-1}$. Previous studies [9], [13] have reported that the radar signatures of crystal reorientation are often associated with intracloud lightning but without ancillary electric field data for the observations presented here it is not possible to determine which of the lightning events were intracloud or cloud-to-ground. However, $1-4 \mathrm{~min}^{-1}$ is well within the flash rates of $0.3-9.3 \mathrm{~min}^{-1}$ observed in Florida thunderstorms [35].

The results show that the specific differential phase shift $\left(k_{D P}\right)$ and LDR at linear polarization can be used to investigate the orientation of ice crystals near the tops of active thunderstorms. These two parameters, which are most sensitive to propagation effects, were the only ones to exhibit cyclic behavior. No orientation related signatures were seen in the S- and X-band reflectivity factors or the differential reflectivity.

A careful interpretation is required in order to separate widespread propagation effects from more local changes in the crystal orientation. The range dependent behavior of the LDR and $k_{D P}$ profiles is critical in the analysis and clearly shows the advantage of radar remote sensing over previously reported results involving only beacon-type propagation tech- niques. The repeated cyclic deviations of $k_{D P}$ and LDR were observed in the Florida storms at heights from 7-14 km AGL. At these heights near cloud top, it is expected that the hydrometeors are pristine ice crystals such as plates or columns. Crystal orientation was observed over ranges of up to $20 \mathrm{~km}$ in a layer $3 \mathrm{~km}$ deep.

LDR may either increase or decrease prior to lightning as the crystals are pulled into alignment, and either show a positive or negative step of as much as $15 \mathrm{~dB}$ after lightning, results consistent with earth-satellite observations [3]. $k_{D P}$ though exhibits a consistent signature of gradually going negative indicating more vertical alignment of the crystals. Average $k_{D P}$ for aligned crystals was typically -0.3 to $-0.5^{\circ}$ $\mathrm{km}^{-1}$, with a minimum of $-0.9^{\circ} \mathrm{km}^{-1}$ observed. These signatures indicate frequent mean orientation angles of greater than $45^{\circ}$ between the semimajor axis and the horizontal and although less common, angles near vertical were inferred for several lightning events. Similar orientations have been reported in observations with circular polarization radar [12], [14].

The reflectivity associated with the Florida observations is 25-40 dBZ. While these values are consistent with theoretical estimates for ice crystals of sizes of 1-1.5 mm (Figs. 1 and 2 ), no orientation signatures were observed in $Z_{S}$ or $Z_{X}$. The absence of reflectivity signatures suggests therefore that the hydrometeors undergoing alignment are smaller than $1 \mathrm{~mm}$ but present in relatively large concentrations.

It is clear that more studies are required to better identify the in situ conditions in which crystals do and do not become aligned, and to better characterize the propagation medium of ice crystals in relation to polarimetric signatures used for detecting orientation changes. The temporal evolution and spatial distribution of the regions of crystal alignment require an improved understanding and suggest areas for future radar observations. All other conditions being equal, a shorter wavelength fully polarimetric radar such as $\mathrm{X}$ band is most applicable to these type of measurements in order to enhance the propagation effect.

\section{ACKNOWLEDGMENT}

The authors would like to thank Dr. P. R. Krehbiel and personnel at the New Mexico Tech Radar for providing invaluable assistance acquiring the observations during the CaPE field project. Prof. V. N. Bringi is gratefully acknowledged for his support. J. Beaver performed the ice crystal scattering computations.

\section{REFERENCES}

[1] B. Vonnegut, "Orientation of ice crystals in the electric field of a thunderstorm," Weather, vol. 20, pp. 310-312, 1965.

[2] D. P. Haworth, N. J. McEwan, and P. A. Watson, "Relationship between atmospheric electricity and microwave radio propagation," Nature, vol. 266, pp. 703-704, 1977.

[3] D. C. Cox and H. W. Arnold, "Observations of rapid changes in the orientation and degree of alignment of ice particles along an earthspace radio propagation path," J. Geophys. Res., vol. 84, no. C8, pp. 5003-5110, 1979.

[4] N. J. McEwan, P. A. Alves, H. W. Poon, and A. W. Dissanayake, "OTS propagation measurements during thunderstorms," Ann. Telecommun. vol. 36, pp. 102-110, 1981. 
[5] P. A. Watson, N. J. McEwan, A. W. Dissanayake, and D. P. Haworth, "Attenuation and cross-polarization measurements at $20 \mathrm{GHz}$ using the ATS-6 satellite with simultaneous radar observations," IEEE Trans. Antennas Propagat., vol. AP-27, pp. 11-17, 1979.

[6] A. Hendry and G. C. McCormick, "Radar observations of the alignment of precipitation particles by electrostatic fields in thunderstorms," $J$. Geophys. Res., vol. 81, pp. 5353-5357, 1976.

[7] G. C. McCormick and A. Hendry, "Radar measurement of precipitationrelated depolarization of thunderstorms," IEEE Trans. Geosci. Electron., vol. GE-17, pp. 142-150, 1979.

[8] A. Hendry and Y. M. M. Antar, "Radar observations of polarization characteristics and lightning-induced realignment of atmospheric ice crystals," Radio Sci., vol. 17, pp. 1243-1250, 1982.

[9] P. R. Krehbiel, W. Rison, S. McCrary, T. Blackman, and M. Brook, "Dual-polarization radar observations of lightning echoes and precipitation alignment at $3 \mathrm{~cm}$ wavelength," in 25th Int. Conf. Radar Meteor., Amer. Meteor. Soc., Boston, MA, 1991, pp. 901-904.

[10] P. R. Krehbiel, T. Chen, S. McCrary, W. Rison, G. Gray, T. Blackman, and M. Brook, "Dual-polarization radar signatures of the potential for lightning in electrified storms," in 9th Int. Conf. Atmos. Elect., St. Petersburg, Russia, 1992, pp. 166-169.

[11] P. Krehbiel, T. Chen, S. McCrary, W. Rison, G. Gray, and M. Brook, "Dual-polarization radar indications of the potential for lightning in storms near Kennedy Space Center, Florida," in 26th Conf. Radar Meteor., Amer. Meteor. Soc., Boston, MA, 1993, pp. 309-311.

[12] _ "The use of dual channel circular-polarization radar observations for remotely sensing storm electrification," Meteor. Atmos. Phys., vol. 58 , pp. 65-82, 1996.

[13] J. Metcalf, "Radar observations of the effects of changing electric fields on the orientations of hydrometeors," Phillips Lab., Air Force Syst. Command, Hanscom AFB, MA, 1992, PL-TR-92-2122, ERP 1100, p. 30.

[14] , "Radar observations of changing orientations of hydrometeors in thunderstorms," J. Appl. Meteor., vol. 34, pp. 757-772, 1995.

[15] V. N. Bringi and A. Hendry, "Technology of polarization diversity radars for meteorology," in Radar in Meteorology, D. Atlas, Ed. Boston: Amer. Meteor. Soc., 1990, pp. 153-190.

[16] T. A. Seliga and V. N. Bringi, "Potential use of radar differential reflectivity measurements at orthogonal polarizations for measuring precipitation," J. Appl. Meteor., vol. 15, pp. 69-76, 1976.

[17] A. R. Jameson, "Relations among linear and circular polarization parameters measured in canted hydrometeors," J. Atmos. Ocean. Technol. vol. 4, pp. $634645,1987$.

[18] V. Chandrasekar, G. R. Grant, and I. J. Caylor, "Auxiliary signal processing system for a multiparameter radar," J. Atmos. Ocean. Technol. vol. 10, pp. 428-431, 1993.

[19] E. A. Mueller, "Calculation procedure for differential propagation phase shift," in 22nd Radar Meteor. Conf., Amer. Meteor. Soc., Boston, MA 1984, pp. 397-399.

[20] V. N. Bringi, V. Chandrasekar, P. Meischner, J. Hubbert, and Y. Golestani, "Polarimetric radar signatures of precipitation at $S$ - and C-bands," Proc. Inst. Elect. Eng., vol. F-138, pp. 109-119, 1991.

[21] M. Sachidananda and D. S. Zrnic, " $Z_{D R}$ measurement considerations for a fast scan capability radar," Radio Sci., vol. 20, pp. 907-922, 1985

[22] C. P. R. Saunders, "A review of thunderstorm electrification processes," J. Appl. Meteor., vol. 32, pp. 642-655, 1993.
[23] E. R. Williams, "Large-scale charge separation in thunderclouds," $J$. Geophys. Res., vol. 90, no. D4, pp. 6013-6025, 1985.

[24] D. A. Burrows and J. C. Stith, "Alignment of ice crystals due to transient electric fields," J. Atmos. Ocean. Technol., vol. 3, pp. 265-272, 1986.

[25] A. J. Weinheimer and A. A. Few, "The electric field alignment of ice particles in thunderstorms," J. Geophys. Res., vol. 92, pp. 14833-14844, 1987

[26] M. A. Uman, The Lightning Discharge. Orlando, FL: Academic, 1987, p. 64.

[27] H. R. Cho, J. V. Irabarne, and W. G. Richards, "On the orientation of ice crystals in a cumulonimbus cloud," J. Atmos. Sci., vol. 38, pp. 1111-1114, 1981.

[28] J. P. Mon, "Backward and forward scattering of microwaves by ice particles: A review," Radio Sci., vol. 17, pp. 953-971, 1982.

[29] J. Vivekanandan, R. Raghavan, and V. N. Bringi, "Polarimetric radar modeling of mixtures of precipitation particles," IEEE Trans. Geosci. Remote Sensing vol. 31, pp. 1017-1030, 1993.

[30] J. Vivekanandan, V. N. Bringi, M. Hagen, and P. Meischner, "Polarimetric radar studies of atmospheric ice particles," IEEE Trans. Geosci. Remote Sensing vol. 32, pp. 1-10, 1994.

[31] I. J. Caylor, V. Chandrasekar, V. N. Bringi, and S. S. Minger, "Multiparameter radar observations of lightning," in 26th Conf. Radar Meteor., Amer. Meteor. Soc., Boston, MA, 1993, pp. 306-308.

[32] M. P. M. Hall, J. W. F. Goddard, and S. M. Cherry, "Identification of hydrometeors and other targets by dual-polarization radar," Radio Sci., vol. 19, pp. 132-140, 1984.

[33] A. R. Jameson, "The interpretation and meteorological application of radar backscatter amplitude ratios at linear polarizations," J. Atmos. Ocean. Technol., vol. 6, pp. 908-919, 1989.

[34] T. A. Seliga, K. Aydin, H. Direskeneli, and V. N. Bringi, "Possible evidence for strong vertical electric fields in thunderstorms from differential reflectivity measurements," in 21st Conf. Radar Meteor., Amer. Met. Soc., Boston, MA, 1983, pp. 500-502.

[35] J. M. Livingston and E. P. Krider, "Electric fields produced by Florida thunderstorms," J. Geophys. Res., vol. 83, no. C1, pp. 385-401, 1978.

I. Jeff Caylor received the B.S. degree in physics and mathematics and the M.S. degree in physics from the New Mexico Institute of Mining and Technology, Socorro, in 1983 and 1985, respectively, and the Ph.D. degree in physics from the University of Manchester Institute of Science and Technology, Manchester, England, in 1989.

He continued research on polarimetric techniques in radar meteorology with the high-resolution Chilbolton radar and later the NCAR CP-2 multiparameter radar. Since 1993, he has been a Staff Scientist at Science Systems and Applications, Inc., providing technical support for the NASA Doppler weather radar which flies on the high altitude ER-2 aircraft. His research interests include polarimetric radar techniques for meteorology, airborne remote sensing, radar data acquisition/processing, and atmospheric electricity.

V. Chandrasekar (S'84-M'87), photograph and biography not available at the time of publication. 